\title{
Genetic Analysis Can Surrogate the Treatment Strategies in Patients with Pancreatic Cancer
}

Dong Uk Kim

Biomedical Research Center, Pusan National University Hospital, Busan, Korea

See "Comprehensive Cancer Panel Sequencing Defines Genetic Diversity and Changes in the Mutational Characteristics of Pancreatic Cancer Patients Receiving Neoadjuvant Treatment" by Kyong-Ah Yoon, et al. on page 683, Vol. 13. No. 6, 2019

Pancreatic cancer is one of the most lethal cancers in the world. The 5-year survival rate has been very low during the last two decades. ${ }^{1}$ This is because the early diagnosis of pancreatic cancer is difficult due to various causes including nonspecific early symptoms, no early diagnostic biomarkers, and no noninvasive and cheaper image modality. Most patients present to the hospital with the advanced stage when they feel more specific symptoms such as abdominal pain and jaundice. Eventually, only 20\% of patients could undergo surgery, and $10 \%$ to $25 \%$ of them experienced recurrence within 1 year. ${ }^{2}$ Therefore, many researchers have tried to find good biomarkers for early diagnosis and early detection of the recurrence. Recent advancement of genetic analysis has shown the development of biomarkers for early diagnosis biomarker in various types of cancers.

In this issue of Gut and Liver, Yoon et al. ${ }^{3}$ evaluated 22 patients who had undergone neoadjuvant chemotherapy and following surgery. They reported that lymphatic invasion was a poor prognostic marker with a median survival of 13 months. Regarding genetic mutation, the high mutational burden was related to the mismatch repair gene mutation, but not related to poor prognosis. KRAS, TP53 and ARID1A genes were the most common somatic mutation in 22 patients. Among those genes, ARID1A was only associated with overall survival. Additionally, highly mutated gene set included the genes which are involved in chromatin modification such as KMT2D, TAF1, ATM, and WHSC1. Their major finding was the genetic change before and after neoadjuvant therapy in seven patients with surgically resected mass. Six of these seven patients showed the lower allelic fraction of KRAS codon 12 mutations in surgically resected specimens than in endoscopic ultrasound (EUS) samples. Four patients of them showed lower allelic fraction of codon 1020 mutation in the WHSC1 gene. According to their data, the authors showed the feasibility of genetic analysis regarding the prognosis and the response to the therapy in pancreatic cancer patients.

There are several comprehensive reports regarding genetic analysis in pancreatic cancer. In The Cancer Genome Atlas (TCGA) study, ${ }^{4}$ they analyzed 146 surgically resected pancreatic cancer of stage I-III. KRAS, TP53, CDKN2A, SMAD4, RNF43, ARID1A, TGFBR2, GNAS, RREB1 and PBRM1 were reported as frequent mutations. They did not report the association between these genes and survival data. However, $42 \%$ of patients had clinically relevant mutations which are eligible for genotypebased target therapy. KRAS mutational alleles were detected in 95\% of samples and mainly located in codon 12 and 61. Therefore, the mutant allele frequency of KRAS could be a common predictive biomarker for the response to therapy in pancreatic cancer.

The European cohort study ${ }^{5}$ showed the association between mutant allelic differences and overall survival in 195 patients with resectable pancreatic cancer. They found 73 nominally most significant single nucleotide polymorphisms (SNPs) which are associated with cancer-related death in European cohort. Of the 73 SNPs, a total of 45 SNPs was also represented in the TCGA cohort. Finally, they identified two common SNPs in regulatory regions of CHI 3 L2 and CD44 genes. These SNPs of CHI3L2 and CD44 genes were related to tumor-associated survival in European cohort after tumor resection. They suggested that these prognostic biomarkers may be used to identify the

\footnotetext{
Correspondence to: Dong Uk Kim

Biomedical Research Center, Pusan National University Hospital, 179 Gudeok-ro, Seo-gu, Busan 49241, Korea

Tel: +82-51-240-7869, Fax: +82-51-244-8180, E-mail: amlm3@hanmail.net pISSN 1976-2283 eISSN 2005-1212 https://doi.org/10.5009/gnl19319

(a) This is an Open Access article distributed under the terms of the Creative Commons Attribution Non-Commercial License (http://creativecommons.org/licenses/by-nc/4.0) which permits unrestricted non-commercial use, distribution, and reproduction in any medium, provided the original work is properly cited.
} 
patient who has invasive biology and need neoadjuvant therapy before tumor resection.

With regard to the genetic analysis in advanced pancreatic cancers, there are fewer studies because the adequate tissue acquisition would be difficult through the ultrasound (US)-guided core needle biopsy (CNB) or EUS-guided fine-needle aspiration/ biopsy (FNA/B). Aguirre et al. ${ }^{6}$ showed the feasibility of realtime genomic characterization in clinical care for advanced pancreatic ductal adenocarcinoma. They developed Panseq protocol which obtained tissues through US-guided CNB or EUS-guided FNA and rapidly moved to genetic analysis such as whole-exome sequencing (WES) and RNA sequencing. Of 79 enrolled patients, WES was analyzed in 71 patients (89\%) and RNA sequencing only in 60 patients (76\%) because other patients showed poor quality of obtained tissue samples. The most frequent somatic mutations were KRAS, TP53, CDKN2A, ARID1A, and TGFBR2. Of 79 patients, only 21 patients (26.6\%) could experience a change of clinical management as a result of genetic analysis. However, they demonstrated that real-time genomic characterization is feasible in the clinical management of advanced pancreatic cancers.

In the early report of the COMPASS trial, ${ }^{7}$ they have tried to perform real-time whole-genome sequencing and RNA sequencing through image-guided CNB and laser capture microdissection of the obtained tissues. Of 63 patients enrolled until June 2017, 62 patients (98\%) received the genomic results within 52 days prior to the first disease assessment. Based on RNA subtype, they suggested GATA6 expression is a potential predictive biomarker for conventional chemotherapy in advanced pancreatic cancer.

The genetic analysis is challenging in pancreatic cancer. The rate of detected mutations is variable due to several reasons. First, the earlier stage of pancreatic cancer may harbor a smaller proportion of mutant alleles. Second, the obtained tissue quality would be lower through US-guide CNB and EUS-guided FNA/ $B$ compared to that of surgically resected tissues. Third, the detection rate of allelic aberration would be lower after treatment even in resected tissues. Fourth, the allelic differences may reflect the ethic differences between Eastern and Western population. Fifth, the differences in the technical protocol could affect the detection of mutant alleles. Nevertheless, Yoon et al. ${ }^{3}$ successfully reported genetic diversity and changes in pancreatic cancer patients receiving neoadjuvant treatment.
In summary, ARID1A gene mutations could be prognostic and KRAS mutational allele frequency would be a potential predictive biomarker in resectable pancreatic cancer. With the advancement of the technical protocol and the accumulation of clinical relevance for genetic analysis in pancreatic cancer, these genetic aberrations could be used as biomarkers to surrogate various treatment options in patients with pancreatic cancer.

\section{CONFLICTS OF INTEREST}

No potential conflict of interest relevant to this article was reported.

\section{ORCID}

Dong Uk Kim

https://orcid.org/0000-0002-7208-7753

\section{REFERENCES}

1. Jung KW, Won YJ, Kong HJ, Lee ES; Community of PopulationBased Regional Cancer Registries. Cancer statistics in Korea: incidence, mortality, survival, and prevalence in 2015. Cancer Res Treat 2018;50:303-316.

2. He J, Ahuja N, Makary MA, et al. 2564 resected periampullary adenocarcinomas at a single institution: trends over three decades. HPB (Oxford) 2014;16:83-90.

3. Yoon KA, Woo SM, Kim YH, et al. Comprehensive cancer panel sequencing defines genetic diversity and changes in the mutational characteristics of pancreatic cancer patients receiving neoadjuvant treatment. Gut Liver 2019;13:683-689.

4. Cancer Genome Atlas Research Network. Integrated genomic characterization of pancreatic ductal adenocarcinoma. Cancer Cell 2017;32:185-203.

5. Dimitrakopoulos C, Vrugt B, Flury R, et al. Identification and validation of a biomarker signature in patients with resectable pancreatic cancer via genome-wide screening for functional genetic variants. JAMA Surg 2019;154:e190484.

6. Aguirre AJ, Nowak JA, Camarda ND, et al. Real-time genomic characterization of advanced pancreatic cancer to enable precision medicine. Cancer Discov 2018;8:1096-1111.

7. Aung KL, Fischer SE, Denroche RE, et al. Genomics-driven precision medicine for advanced pancreatic cancer: early results from the COMPASS trial. Clin Cancer Res 2018;24:1344-1354. 